\section{Ditching causality}

\author{
Peter Holland
}

Quantum Mechanics: Historical Contingency and the Copenhagen Hegemony. By James T. Cushing. University of Chicago Press: 1994. Pp. 317. \$65, £51 (hbk); \$27, £21.50 (pbk).

The Mystery of the Quantum World, second edition. By Euan Squires. Institute of Physics Publishing: 1994. Pp. 191. £45, $\$ 90$ (hbk); £12.95, \$24.50 (pbk).

An Interpretive Introduction to Quantum Field Theory. By Paul Teller. Princeton University Press: 1995. Pp. 176. \$35, £23.50.

THE foundations of quantum mechanics as an independent subject provides a salutary lesson for those who see scientific development as driven by internal dynamics divorced from the social context of scientists. For what we today take for granted as a respectable and thriving field was, not so long ago, a dangerous move for a career-minded scientist. Its existence is due in no small measure to the struggles of a handful of dissidents, including some of this century's finest scientists (Einstein, de Broglie, Schrödinger), who wanted physics to be based on a clear and consistent ontology. They believed, as John Bell put it, "that vagueness, subjectivity and indeterminism are not forced on us by experimental facts, but by deliberate theoretical choice". They raised questions that the orthodoxy in theoretical physics, in place by the late 1920 s, did not so much answer as ignore. As pointed out in James Cushing's fine book, one reason the dissenters were unable to establish their alternative was that they were divided in their views of what a desirable ontology should be, and so could not mount a coherent challenge to a united opposition.

The subject of Cushing's book is how a point of view that denied the possibility of causal space-time representations of atomic phenomena, the Copenhagen interpretation developed principally by Bohr, gained ascendancy over equally viable (in the sense of empirical adequacy) descriptions that did employ such representations. He recounts the events that led first to the crushing of de Broglie's tentative proposals for a space-time model in 1927 and then to the marginalization of David Bohm's fully fledged theory in 1952. Cushing argues persuasively that an historical error has been made in eschewing causal models and illustrates his case by comparing the quality of the explanations provided by the two world views for typical effects such as twoslit interference. His thesis is that Copenhagen triumphed not because it had more compelling technical arguments but because it got in first, became entrenched and used the dead-weight of authority to see off all competitors.

It was a fortuitous victory. Cushing speculates how the story might have turned out had the order of certain ("historically contingent") events been different. Historians may see this as an idle exercise but the author's aim is to show that scientific arguments alone did not justify the wholesale ditching of causality as a goal of physics. This decision, whatever its merits, did not flow from facts forced on us by 'nature' but from extra-scientific pressures. Along the way, Cushing reveals important but little known material, such as an unpublished hidden-variable interpretation of Einstein.

I am not sure Cushing has got to the bottom of this story, for Bohm's ideas have been anathema even to physicists who have sustained a critical attitude in questions of interpretation. Renowned scientists have spent years promoting the bizarre suggestion that the Universe 'splits' whenever a choice is available for the future behaviour of an electron, in preference to Bohm's simple notion that an electron moves along a track in space. Another important cultural issue not addressed is why it should be now, after 40 years of neglect, that many workers are attracted to Bohm's theory. But the author is to be applauded for recognizing the need for this analysis and for producing a sustained and well written account that will undoubtedly stimulate others to look at other aspects of a remarkable story, especially at the crucial role of dissidence in scientific culture.

Euan Squires's well known book is one of the few that successfully straddle the huge gulf between formal texts and traditional popularizations of quantum mechanics. It is aimed at the general reader but I suspect its natural place is as an adjunct to an undergraduate course. In that context it will play a valuable role in showing students that the physical interpretation of the techniques they are learning is a matter of continuing and unresolved debate. The author gives clear descriptions of the problem of quantum reality, the role of consciousness, and nonlocality, including an accessible account of Bell's theorem. There are helpful anecdotes, including an account of his own experience as a student in the 1950s when discussion of these issues was frowned upon. Squires brings the first edition up to date through an additional chapter on recent developments in collapse theories, the Bohm model and Lorentz invariance. It is a pity it was not possible to integrate this material into the main text, for Squires's own views on these topics, to which he himself has made important contributions, have moved on since the previous edition.

It is fair to say that our comprehension of quantum field theory is more slender even than that of elementary quantum mechanics. Paul Teller faces up to this issue by analysing the meaning of the terms used in traditional textbook treatments of field theory. (He unfortunately does not discuss the currently popular functional techniques that are pregnant with possibilities for imaginative new interpretations.) What he does do is examine how the concepts of 'particle' and 'field' are to be understood and asks whether they can be smoothly connected. He discusses how the notion of an 'operator-valued field' is related to our customary concept of a field and shows, correctly I think, that a direct association of the two is misleading. Although questions may be raised about the physical picture implied by his use of the 'propensity' concept, I believe this is a valuable book. Indeed, the specific interpretative proposals he makes could have an influence on hidden-variable theories. The author expresses scepticism about hidden-variable theories, but if he had adopted a definition more in accord with their actual characteristics he might have found fruitful overlap between the two programmes. At the risk of labouring the point, Bohm's theory has many interesting things to say about spacetime pictures of quantum fields, and it would be interesting to explore how it impinges on Teller's considerations.

These three books reflect a welcome trend towards a reassessment of the notions of meaning and visualization in contemporary science. Scientists from other disciplines would be surprised to learn how much effort has been expended in expunging causal imagery from physics. Yet for all their protests to the contrary, even the most committed Copenhagen supporter privately employs images of the micro-domain because these are indispensable when applying quantum mechanics in unfamiliar situations. It is a merit of Bohm and others that they were honest about this, and sought to go beyond the odd amalgam of classical ideas espoused by Bohr to find a more satisfactory iconography.

Peter Holland is at the School of Interdisciplinary Sciences, University of the West of England, Bristol BS16 1QY, UK.

\section{Corrections}

In Peter Knight's review of Optical Coherence and Quantum Optics by Leonard Mandel and Emil Wolf (Nature 378, 348; 1995), a line was inadvertently missed out in the editing, leading to the incorrect statement that Wolf is an experimentalist. The fourth and fifth sentences of the second paragraph should in fact read: "Wolf was the originator of many of our fundamental ideas of coherence, and with Mandel he pioneered much of our understanding of photon statistics. Mandel was the founder of the experimental study of nonclassical light and the first to generate laboratory sources of light of totally quantum origin such as antibunched light and the correlated twin beams of light from parametric amplifiers."

In Peter J. Bowler's review of Gregor Mendel by Vitezslav Orel (Nature 378, 100; 1995), Brno is described as being in Austria rather than in the Czech Republic. Both mistakes were made in the editorial office of Nature. Our apologies. 